\title{
Outcome Prediction of Acute Kidney Injury Biomarkers at Initiation of Dialysis in Critical Units
}

\author{
Vin-Cent $\mathrm{Wu}^{1}{ }^{(1)}$, Chih-Chung Shiao ${ }^{2,3}{ }^{(1)}$, Nai-Hsin Chi ${ }^{4}$, Chih-Hsien Wang ${ }^{4}$, \\ Shih-Chieh Jeff Chueh ${ }^{5}$, Hung-Hsiang Liou ${ }^{6}$, Herbert D. Spapen ${ }^{7, *}$, Patrick M. Honore ${ }^{8, *}$ \\ and Tzong-Shinn Chu ${ }^{1,9, *}$ \\ 1 Division of Nephrology, National Taiwan University Hospital, No. 7 Chung-Shan South Road, \\ Zhong-Zheng District, Taipei 100, Taiwan; q91421028@ntu.edu.tw \\ 2 Division of Nephrology, Department of Internal Medicine, Saint Mary's Hospital Luodong, \\ No. 160 Chong-Cheng South Road, Loudong, Yilan 265, Taiwan; chungyy2001@yahoo.com.tw \\ 3 Saint Mary's Junior College of Medicine, Nursing and Management College, No. 100, Ln. 265, Sec. 2, \\ Sanxing Rd., Sanxing Township, Yilan 266, Taiwan \\ 4 Surgery Department, National Taiwan University Hospital, No. 7 Chung-Shan South Road, \\ Zhong-Zheng District, Taipei 100, Taiwan; chinaihsin@ntuh.gov.tw (N.-H.C.); wchemail@gmail.com (C.-H.W.) \\ 5 Glickman Urological and Kidney Institute, Cleveland Clinic Lerner College of Medicine, Cleveland Clinic, \\ Cleveland, 9500 Euclid Ave., Cleveland, OH 44195, USA; jeffchueh@gmail.com \\ 6 Division of Nephrology, Department of Internal Medicine, Hsin-Jen Hospital, Dialysis Center, \\ Hsin-Ren Clinics, No. 395, Chung-Shan Road, New Taipei City 231, Taiwan; hh258527@ms23.hinet.net \\ 7 ICU Department, Universitair Ziekenhuis Brussel, Vrije Universiteit Brussel, 101, Laarbeeklaan, \\ 1090 Jette, Belgium \\ 8 ICU Department, CHU Brugmann University Hospital, 4 Place Arthur Van Gehucthen, 1020 Brussels, Belgium \\ 9 NSARF Group (National Taiwan University Hospital Study Group of ARF), Taipei 100, Taiwan \\ * Correspondence: herbert.spapen@uzbrussel.be (H.D.S.); Patrick.Honore@CHU-Brugmann.be (P.M.H.); \\ tschu@ntu.edu.tw (T.-S.C.)
}

Received: 18 July 2018; Accepted: 3 August 2018; Published: 6 August 2018

\begin{abstract}
The ideal circumstances for whether and when to start RRT remain unclear. The outcome predictive ability of acute kidney injury (AKI) biomarkers measuring at dialysis initializing need more validation. This prospective, multi-center observational cohort study enrolled 257 patients with AKI undergoing renal replacement therapy (RRT) shortly after admission. At the start of RRT, blood and urine samples were collected for relevant biomarker measurement. RRT dependence and all-cause mortality were recorded up to 90 days after discharge. Areas under the receiver operator characteristic (AUROC) curves and a multivariate generalized additive model were applied to predict outcomes. One hundred and thirty-five (52.5\%) patients died within 90 days of hospital discharge. Plasma c-terminal FGF-23 (cFGF-23) had the best discriminative ability (AUROC, 0.687) as compared with intact FGF-23 (iFGF-23) (AUROC, 0.504), creatinine-adjusted urine neutrophil gelatinase-associated lipocalin (AUROC, 0.599), and adjusted urine CFGF-23 (AUROC, 0.653) regardless whether patients were alive or not on day 90. Plasma cFGF-23 levels above $2050 \mathrm{RU} / \mathrm{mL}$ were independently associated with higher 90-day mortality (HR 1.76, $p=0.020$ ). Higher cFGF-23 levels predicted less weaning from dialysis in survivors (HR, $0.62, p=0.032)$, taking mortality as a competing risk. Adding CFGF-23 measurement to the AKI risk predicting score significantly improved risk stratification and 90-day mortality prediction (total net reclassification improvement $=0.148 ; p=0.002$ ). In patients with AKI who required RRT, increased plasma cFGF-23 levels correlated with higher 90-day overall mortality after discharge and predicted worse kidney recovery in survivors. When coupled to the AKI risk predicting score, cFGF-23 significantly improved mortality risk prediction. This observation adds evidence that cFGF-23 could be used as an optimal timing biomarker to initiate RRT.
\end{abstract}


Keywords: acute kidney injury; biomarker; fibroblast growth factor-23; kidney injury molecule-1; mortality; neutrophil gelatinase-associated lipocalin; renal replacement therapy

\section{Background}

Renal replacement therapy (RRT) is life-saving in patients with acute kidney injury (AKI) but is not devoid of serious complications and severe adverse events [1]. Patients who, even temporarily, require RRT also may develop more frequently long-term or end-stage renal disease (ESRD) and have a higher mortality risk [2]. The need for and the optimal timing to initiate RRT are crucial yet unresolved issues $[1,3]$.

Nephrologists continuously look out for kidney specific biomarkers that assist in fine-tuning of diagnosis, treatment, and prognosis of AKI [4]. Few biomarkers were validated as outcome-specific biomarkers in critically ill patients at initiation of RRT. Urine neutrophil gelatinase-associated lipocalin (NGAL) was one of the first biomarkers to be validated for predicting short-term mortality in patients with advanced AKI [5] and recently became part of the indicators to decide early start of dialysis [6]. Interleukin-18 (IL-18) at the commencement of dialysis could also predict hospital mortality in critically ill patients [7]. Adding plasma interleukin-8 to a parsimonious clinical model (i.e., age, mean arterial pressure, mechanical ventilation, and bilirubin) augmented prediction of renal recovery and AKI mortality compared with using only the clinical variables [8].

Fibroblast growth factor 23 (FGF-23), a peptide initially recognized for its phosphaturic role in rare genetic or acquired hypophosphatemia disorders [9], is one of the most recently proposed kidney biomarkers. FGF-23 acts as a hormone that significantly influences phosphate, vitamin D, and bone mineral homeostasis [10]. Several research groups have proposed cFGF-23 as a biomarker for predicting early occurrence of AKI, evaluating prognosis of chronic kidney disease (CKD), and estimating cardiovascular morbidity and mortality [11-15].

An important area of AKI research particularly focuses on reinforcing current dialysis requiring AKI by adding measurement of (a) sensitive biomarker (s) to assess the impact of RRT on relevant patient outcome variables. Within this perspective, we designed a study to evaluate the predictive capacity of various structural and functional kidney biomarkers (including the novel markers cFGF-23 and iFGF-23) and disease severity scores, measured at initiation of RRT, on survival and renal function recovery in a cohort of AKI patients.

\section{Methods}

\subsection{Registration of Clinical Trials}

This study was approved by the University's Institutional Review Board (201409024RINB in National Taiwan University Hospital, 01-X16-059 in Buddhist Tzu Chi General Hospital, and TYGH104007 in Taoyuan General Hospital) and written informed consent was obtained from all subjects participating in the trial. The trial was registered prior to patient enrollment at clinicaltrials.gov (NCT01503710, Principal investigator: V.-C.W, Date of registration: 28 February 2012).

\subsection{Study Population}

The study was conducted by the National Taiwan University Study Group on Acute Renal Failure (NSARF) and based on a prospectively created AKI database [16-20]. From August 2011 until January 2015, 257 AKI patients who required RRT after intensive care unit (ICU) admission were prospectively enrolled. Exclusion criteria included: age $<18$ years, previous nephrectomy, renal transplantation or RRT treatment, ICU or hospital length of stay of respectively $<2$ days and $>180$ days during the index hospitalization, and AKI caused by urologic surgery induced injury, vasculitis, obstruction, glomerulonephritis, interstitial nephritis, hemolytic uremic syndrome, or thrombotic thrombocytopenic purpura. 


\subsection{Data Collection}

Baseline characteristics, including demographic data, co-morbidities, the cause of AKI. For the risk prediction before initializing dialysis, the individual AKI risk predicting score was calculated [21]. The worst physiological values and biochemical data on the index day were recorded.

Baseline serum creatinine ( $(\mathrm{Cr})$ was the nadir value obtained after the previous admission in those who had more than one admission within 1 year before the index admission, or estimated with the Modification of Diet in Renal Disease equation (assuming an average eGFR of $75 \mathrm{~mL} / \mathrm{min} / 1.73 \mathrm{~m}^{2}$ ) [22]. Peak sCr was defined as the highest sCr before RRT initiation in the ICU. Indication for dialysis and organ failure were defined as previously reported $[16,23,24]$ (Supplemental Data file).

RRT modalities in each patient were initially chosen by the attending physician and adapted according to hemodynamic evaluation and evolution by the critical care nephrologist (Supplemental Data file).

\subsection{Measurements of Kidney Biomarkers}

The urine samples, collected in separate polypropylene tubes containing sodium azide at dialysis initiation, were stored at $-80^{\circ} \mathrm{C}$ until required. Each specimen was centrifuged $\left(800 \times g\right.$ at $4{ }^{\circ} \mathrm{C}$ for $5 \mathrm{~min}$ ) and the supernatant was collected for ELISA analysis.

Kidney biomarker levels were assessed with a human FGF-23 C-terminal/intact-terminal ELISA kit (Immutopics; San Clemente, CA, USA), a human KIM-1, and a lipocalin-2/NGAL ELISA kit (R\&D Systems, Inc., Minneapolis, MN, USA).

The cFGF-23 and iFGF-23 values were expressed in relative units $(\mathrm{RU}) / \mathrm{mL}$ and $\mathrm{pg} / \mathrm{mL}$, respectively. The coefficient of variation was $4.4 \%$ for iFGF-23 and $4.0 \%$ for cFGF-23. The lower limits for detection of cFGF-23, iFGF-23, KIM-1 and NGAL were $0.156 \mathrm{RU} / \mathrm{mL}, 0.2 \mathrm{pg} / \mathrm{mL}$, $0.046 \mathrm{ng} / \mathrm{mL}$, and $0.04 \mathrm{ng} / \mathrm{mL}$, respectively were completed as described by the manufacturer's protocol and performed in duplicate. 1,25 di hydroxyvitamin D was measured using DiaSorin radioimmunoassay assays kit (Stillwater, MN, USA) and total 25-hydroxyvitamin D was measured using an electro-chemiluminescence (Elecsys ${ }^{\circledR}$ Vitamin D total, Cobas, Roche@). Urine creatinine levels were measured with the Jaffe assay, with standardization of the isotope dilution mass spectrometry traceable reference.

\subsection{Outcome Definitions}

Primary clinical endpoints were 90-day mortality after hospital discharge and dialysis dependency at 90 days in survivors. Secondary end-points included a 90-day composite outcome (ongoing dialysis or 90-day mortality after discharge), in-hospital mortality, and a composite outcome at discharge (ongoing dialysis or mortality at discharge). All patients were followed until death or for a time span exceeding 90 days after discharge, whichever occurred first. Successful withdrawal from dialysis was defined as surviving without dialysis at the end of study.

\subsection{Statistical Analysis}

All the univariate significant and non-significant relevant covariates, including age, sex, baseline comorbidities, indication for dialysis, etiology of AKI, kidney function profile (e.g., baseline eGFR and candidate biomarkers, candidate biomarkers and SOFA score at dialysis initiation, dialysis modality, and some of their interactions were put on the variable lists to be selected (Table 1). Two-sample student's $t$-test was used to analyze continuous data and $\chi^{2}$ test or Fisher's exact test was used to analyze categorical data. The accumulated hazard ratio was modeled by Cox regression models and adjusted for the covariates for the outcomes of interest (Supplemental Data file). The significance levels for entry (SLE) and for stay (SLS) were set to 0.15 for being conservative. Then, with the aid of substantive knowledge, the best candidate final logistic regression model was identified manually by dropping the covariates with $p>0.05$ one at a time until all regression coefficients were significantly different from 0. 
Table 1. Clinical characteristics of patient grouped by 90 days outcome.

\begin{tabular}{|c|c|c|c|c|c|c|c|}
\hline & All & 90-Day Survival & 90-Day Mortality & $p$ & $\begin{array}{c}\text { 90-Day Composite } \\
\text { Outcome }(-)\end{array}$ & $\begin{array}{l}\text { 90-Day Composite } \\
\text { Outcome (+) }\end{array}$ & $p$ \\
\hline & $(n=257)$ & $(n=122)$ & $(n=135)$ & & $(n=76)$ & $(n=181)$ & \\
\hline \multicolumn{8}{|l|}{ Patient characteristics } \\
\hline Age & $65.7 \pm 16.6$ & $63.4 \pm 16.0$ & $67.8 \pm 16.9$ & 0.035 & $61.3 \pm 17.5$ & $67.6 \pm 15.9$ & 0.005 \\
\hline Gender (male (\%)) & $167(65.0 \%)$ & $82(67.2 \%)$ & $85(63.0 \%)$ & 0.514 & $54(71.1 \%)$ & $113(62.4 \%)$ & 0.200 \\
\hline Baseline creatinine (mg/dL) & $2.0 \pm 1.6$ & $2.5 \pm 1.9$ & $1.5 \pm 1.1$ & $<0.001$ & $1.8 \pm 1.3$ & $2.1 \pm 1.7$ & 0.220 \\
\hline eGFR (MDRD) $\left(\mathrm{mL} / \mathrm{min} / 1.73 \mathrm{~m}^{2}\right)$ & $55.6 \pm 41.0$ & $48.3 \pm 44.2$ & $62.2 \pm 36.9$ & 0.006 & $63.3 \pm 47.6$ & $52.3 \pm 37.6$ & 0.428 \\
\hline \multicolumn{8}{|l|}{ Co-morbidities } \\
\hline Diabetes mellitus & $115(44.7 \%)$ & $61(50.0 \%)$ & $54(40.0 \%)$ & 0.132 & $33(43.4 \%)$ & $82(45.3 \%)$ & 0.891 \\
\hline Cirrhosis & $9(3.5 \%)$ & $3(2.5 \%)$ & $6(4.4 \%)$ & 0.505 & $2(2.6 \%)$ & $7(3.9 \%)$ & 0.999 \\
\hline COPD & $15(5.8 \%)$ & $5(4.1 \%)$ & $10(7.4 \%)$ & 0.297 & $5(6.6 \%)$ & $10(5.5 \%)$ & 0.777 \\
\hline CAD & $54(21.0 \%)$ & $24(19.7 \%)$ & $30(22.2 \%)$ & 0.648 & $18(23.7 \%)$ & $36(19.9 \%)$ & 0.505 \\
\hline CVA & $24(9.3 \%)$ & $9(7.4 \%)$ & $15(11.1 \%)$ & 0.392 & $4(5.3 \%)$ & $20(11.0 \%)$ & 0.166 \\
\hline Congestive heart failure & & & & 0.683 & & & 0.780 \\
\hline 0 & $67(26.1 \%)$ & $33(27.0 \%)$ & $34(25.2 \%)$ & & $19(25.0 \%)$ & $48(26.5 \%)$ & \\
\hline I & $100(38.9 \%)$ & $43(35.2 \%)$ & $57(42.2 \%)$ & & $28(36.8 \%)$ & $72(39.8 \%)$ & \\
\hline II & $51(19.8 \%)$ & $24(19.7 \%)$ & $27(20.0 \%)$ & & $14(18.4 \%)$ & $37(20.4 \%)$ & \\
\hline III & $31(12.1 \%)$ & $17(13.9 \%)$ & $14(10.4 \%)$ & & $12(15.8 \%)$ & $19(10.5 \%)$ & \\
\hline \multicolumn{8}{|l|}{ Laboratory data at ICU admission } \\
\hline BUN (mg/dL) & $48.0 \pm 33.5$ & $58.1 \pm 34.5$ & $38.9 \pm 29.9$ & $<0.001$ & $48.9 \pm 36.6$ & $47.7 \pm 32.2$ & 0.783 \\
\hline $\mathrm{pH}$ & $7.4 \pm 0.1$ & $7.4 \pm 0.8$ & $7.4 \pm 0.1$ & 0.659 & $7.4 \pm 0.1$ & $7.4 \pm 0.1$ & 0.612 \\
\hline $\mathrm{FiO}_{2}$ & $0.5 \pm 0.2$ & $0.5 \pm 0.2$ & $0.5 \pm 0.2$ & 0.916 & $5 \pm 0.2$ & $0.5 \pm 0.2$ & 0.218 \\
\hline $\mathrm{SBP}(\mathrm{mmHg})$ & $121.0 \pm 28.4$ & $129.8 \pm 28.8$ & $113.0 \pm 25.6$ & $<0.001$ & $126.0 \pm 25.6$ & $118.8 \pm 29.3$ & 0.063 \\
\hline GCS & $11.9 \pm 4.2$ & $12.3 \pm 4.0$ & $11.6 \pm 4.4$ & 0.164 & $11.9 \pm 4.1$ & $11.9 \pm 4.3$ & 0.948 \\
\hline SOFA & $8.9 \pm 3.5$ & $8.3 \pm 3.1$ & $9.5 \pm 3.7$ & 0.008 & $8.7 \pm 3.4$ & $9.1 \pm 3.6$ & 0.410 \\
\hline APACHE II & $16.3 \pm 6.2$ & $15.6 \pm 6.0$ & $9.5 \pm 3.8$ & 0.094 & $15.0 \pm 6.4$ & $16.9 \pm 6.0$ & 0.025 \\
\hline MODS & $5.9 \pm 3.7$ & $5.5 \pm 3.4$ & $6.4 \pm 3.8$ & 0.040 & $5.7 \pm 3.3$ & $6.0 \pm 3.8$ & 0.507 \\
\hline
\end{tabular}


Table 1. Cont

\begin{tabular}{|c|c|c|c|c|c|c|c|}
\hline & All & 90-Day Survival & 90-Day Mortality & $p$ & $\begin{array}{c}\text { 90-Day Composite } \\
\text { Outcome }(-)\end{array}$ & $\begin{array}{l}\text { 90-Day Composite } \\
\text { Outcome (+) }\end{array}$ & $p$ \\
\hline & $(n=257)$ & $(n=122)$ & $(n=135)$ & & $(n=76)$ & $(n=181)$ & \\
\hline \multicolumn{8}{|l|}{ Etiology of AKI } \\
\hline Shock & $150(58.4 \%)$ & $56(5.9 \%)$ & $94(69.6)$ & $<0.001$ & $40(52.6 \%)$ & $110(60.8 \%)$ & 0.268 \\
\hline Sepsis & $98(38.1 \%)$ & $26(23.8 \%)$ & $69(51.1 \%)$ & $<0.001$ & $22(28.9 \%)$ & $76(42.0 \%)$ & 0.067 \\
\hline Drug-induced & $3(1.2 \%)$ & $0(0 \%)$ & $3(2.2 \%)$ & 0.249 & $0(0 \%)$ & $3(1.7 \%)$ & 0.557 \\
\hline Rhabdomyolysis & $9(3.5 \%)$ & $5(4.1 \%)$ & $4(3.0 \%)$ & 0.740 & $4(5.3 \%)$ & $5(2.8 \%)$ & 0.457 \\
\hline Pigmentation & $6(2.3 \%)$ & $4(3.3 \%)$ & $2(1.5 \%)$ & 0.427 & $4(5.3 \%)$ & $2(1.1 \%)$ & 0.065 \\
\hline Contrast & $37(14.4 \%)$ & $22(18.0 \%)$ & $15(11.1 \%)$ & 0.154 & $13(17.1 \%)$ & $24(13.3 \%)$ & 0.440 \\
\hline Other & $26(10.1 \%)$ & $16(13.1 \%)$ & $10(7.4 \%)$ & 0.150 & $7(9.2 \%)$ & $19(10.5 \%)$ & 0.825 \\
\hline \multicolumn{8}{|l|}{ At initiating dialysis } \\
\hline Admission to dialysis (days) & $40.3 \pm 27.1$ & $42.0 \pm 31.8$ & $37.1 \pm 47.5$ & 0.335 & $45.8 \pm 33.9$ & $36.8 \pm 43.1$ & 0.106 \\
\hline Mechanical Ventilation & $185(72.0 \%)$ & $74(60.7 \%)$ & $111(82.2 \%)$ & $<0.001$ & $49(64.5 \%)$ & $136(75.1 \%)$ & 0.095 \\
\hline Emergency Surgery & $100(38.9 \%)$ & $49(40.2 \%)$ & $51(37.8 \%)$ & 0.703 & $33(43.4 \%)$ & $67(37.0 \%)$ & 0.400 \\
\hline IABP & $27(10.5 \%)$ & $10(8.2 \%)$ & $17(12.6 \%)$ & 0.310 & $7(9.2 \%)$ & $20(11.0 \%)$ & 0.824 \\
\hline Urine output (mL/24 h) & $591.7 \pm 790.3$ & $750.3 \pm 1013.0$ & $448.3 \pm 472.1$ & 0.002 & $869.7 \pm 1188.7$ & $474.9 \pm 503.1$ & $<0.001$ \\
\hline AKI risk prediction score & $22.6 \pm 6.9$ & $20.2 \pm 6.5$ & $24.9 \pm 6.5$ & $<0.001$ & $20.8 \pm 6.4$ & $23.4 \pm 7.0$ & 0.004 \\
\hline Body weight (kg) & $66.8 \pm 14.3$ & $68.6 \pm 15.9$ & $67.8 \pm 16.9$ & 0.055 & $70.0 \pm 15.9$ & $65.5 \pm 13.4$ & 0.021 \\
\hline IE & $8.2 \pm 15.0$ & $4.7 \pm 8.3$ & $11.3 \pm 18.7$ & $<0.001$ & $5.24 \pm 9.32$ & $9.43 \pm 16.75$ & 0.041 \\
\hline SOFA & $10.9 \pm 3.9$ & $9.1 \pm 3.2$ & $12.6 \pm 3.8$ & $<0.001$ & $9.4 \pm 3.3$ & $11.6 \pm 4.0$ & $<0.001$ \\
\hline APACHE II & $17.8 \pm 6.4$ & $15.6 \pm 5.4$ & $19.8 \pm 6.7$ & $<0.001$ & $15.5 \pm 5.7$ & $18.7 \pm 6.5$ & $<0.001$ \\
\hline MODS & $8.1 \pm 4.1$ & $6.5 \pm 3.7$ & $9.5 \pm 3.9$ & $<0.001$ & $7.0 \pm 3.6$ & $8.6 \pm 4.2$ & 0.005 \\
\hline Phosphate (mg/dL) & $4.5 \pm 1.7$ & $4.8 \pm 1.6$ & $4.3 \pm 1.8$ & 0.085 & $4.8 \pm 1.5$ & $4.4 \pm 1.8$ & 0.333 \\
\hline $25 \mathrm{OH}$ Vit D, ng/mL & $11.7 \pm 5.6$ & $10.8 \pm 5.5$ & $12.9 \pm 5.9$ & 0.471 & $11.2 \pm 7.1$ & $12.0 \pm 5.2$ & 0.812 \\
\hline $1,25 \mathrm{diOH}$ Vit $\mathrm{D}, \mathrm{pg} / \mathrm{mL}$ & $27.3 \pm 6.5$ & $25.5 \pm 6.4$ & $29.7 \pm 6.4$ & 0.545 & $28.9 \pm 6.8$ & $26.6 \pm 6.6$ & 0.545 \\
\hline
\end{tabular}


Table 1. Cont.

\begin{tabular}{|c|c|c|c|c|c|c|c|}
\hline & All & 90-Day Survival & 90-Day Mortality & $p$ & $\begin{array}{c}\text { 90-Day Composite } \\
\text { Outcome }(-)\end{array}$ & $\begin{array}{l}\text { 90-Day Composite } \\
\text { Outcome (+) }\end{array}$ & $p$ \\
\hline & $(n=257)$ & $(n=122)$ & $(n=135)$ & & $(n=76)$ & $(n=181)$ & \\
\hline \multicolumn{8}{|l|}{ Kidney function marker } \\
\hline BUN (mg/dL) & $82.4 \pm 47.2$ & $82.7 \pm 51.5$ & $82.5 \pm 45.4$ & 0.922 & $82.3 \pm 51.5$ & $82.5 \pm 45.4$ & 0.978 \\
\hline Creatinine (mg/dL) & $2.0 \pm 1.6$ & $4.1 \pm 2.2$ & $4.2 \pm 2.4$ & 0.745 & $4.1 \pm 2.2$ & $4.2 \pm 2.4$ & 0.745 \\
\hline Urine NGAL (ng/mL) & $197.5 \pm 85.3$ & $191.0 \pm 93.3$ & $203.5 \pm 77.1$ & 0.254 & $189.2 \pm 97.6$ & $201.0 \pm 79.7$ & 0.330 \\
\hline Urine NGAL/Cre & $6.9 \pm 11.1$ & $6.8 \pm 12.5$ & $6.9 \pm 9.7$ & 0.912 & $5.0 \pm 6.9$ & $7.7 \pm 12.4$ & 0.085 \\
\hline Urine KIM1 (ng/mL) & $6.0 \pm 5.8$ & $5.8 \pm 5.8$ & $6.2 \pm 5.8$ & 0.529 & $5.9 \pm 6.5$ & $5.7 \pm 5.4$ & 0.139 \\
\hline Urine KIM1/Cre & $0.1 \pm 0.2$ & $0.1 \pm 0.2$ & $0.1 \pm 0.1$ & 0.993 & $0.1 \pm 0.1$ & $0.1 \pm 0.2$ & 0.699 \\
\hline Urine cFGF-23/Cre & $877.4 \pm 994.3$ & $671.4 \pm 924.9$ & $1063.5 \pm 1021.2$ & $<0.001$ & $699.1 \pm 1015.0$ & $952.2 \pm 978.6$ & 0.062 \\
\hline Plasma iFGF-23 (pg/mL) & $304.2 \pm 468.0$ & $395.1 \pm 635.6$ & $269.0 \pm 385.2$ & 0.265 & $320.4 \pm 551.8$ & $300.2 \pm 449.5$ & 0.875 \\
\hline Plasma cFGF-23 (RU/mL) & $2630.1 \pm 2259.5$ & $1926.7 \pm 1745.4$ & $3265.9 \pm 2479.0$ & $<0.001$ & $1925.3 \pm 1917.3$ & $2926.1 \pm 2330.0$ & 0.001 \\
\hline \multicolumn{8}{|l|}{ Indication for dialysis } \\
\hline Azotemia & $123(47.9 \%)$ & $58(47.5 \%)$ & $65(48.1 \%)$ & 0.999 & $32(42.1 \%)$ & $91(50.3 \%)$ & 0.274 \\
\hline Fluid overload & $111(43.2 \%)$ & $51(41.8 \%)$ & $60(44.4 \%)$ & 0.706 & $30(39.5 \%)$ & $81(44.8 \%)$ & 0.491 \\
\hline Electrolyte disorders & $18(7.0 \%)$ & $10(8.2 \%)$ & $18(5.9 \%)$ & 0.626 & $7(9.2 \%)$ & $11(6.1 \%)$ & 0.423 \\
\hline Metabolic acidosis & $46(17.9 \%)$ & $17(13.9 \%)$ & $29(21.5 \%)$ & 0.143 & $11(14.5 \%)$ & $35(19.3 \%)$ & 0.380 \\
\hline Oliguria & $166(64.6 \%)$ & $69(56.6 \%)$ & $97(71.9 \%)$ & 0.013 & $46(56.6 \%)$ & $123(68.0 \%)$ & 0.088 \\
\hline Uremic encephalopathy & $12(4.7 \%)$ & $9(7.4 \%)$ & $3(2.2 \%)$ & 0.074 & $6(7.9 \%)$ & $6(3.3 \%)$ & 0.191 \\
\hline \multicolumn{8}{|l|}{ Dialysis modality } \\
\hline $\mathrm{CVVH}$ & $62(21.1 \%)$ & $16(13.1)$ & $46(34.1 \%)$ & $<0.001$ & $15(19.7 \%)$ & $47(26.0 \%)$ & 0.296 \\
\hline IHD & $62(29.2 \%)$ & $47(38.5 \%)$ & $28(20.7 \%)$ & & $27(35.5 \%)$ & $48(26.5 \%)$ & \\
\hline SLED & $120(46.7 \%)$ & $59(48.5 \%)$ & $61(45.2 \%)$ & & $34(44.7 \%)$ & $86(47.5 \%)$ & \\
\hline \multicolumn{8}{|l|}{ Relevant outcome parameters } \\
\hline Hospital length of stay (days) & $54.7 \pm 50.4$ & $52.3 \pm 41.1$ & $56.9 \pm 57.6$ & 0.459 & $59.0 \pm 46.3$ & $52.9 \pm 52.0$ & 0.383 \\
\hline Duration of hospital dialysis (days) & $82.4 \pm 60.7$ & $42.0 \pm 31.8$ & $37.1 \pm 47.5$ & 0.335 & $45.8 \pm 33.9$ & $36.8 \pm 43.1$ & 0.745 \\
\hline
\end{tabular}

Abbreviations: AKI, acute kidney injury: APACHE; Acute Physiology and Chronic Health Evaluation, BMI, body mass index; CABG, coronary artery bypass graft; BUN, blood urea nitrogen; $\mathrm{COPD}$, chronic obstructive pulmonary disease; $\mathrm{CPB}$, cardiopulmonary bypass; Cre, creatinine; CVA, cerebrovascular accident; $\mathrm{CVVH}$, continuous venovenous hemofiltration; eGFR, estimated glomerular filtration rate; FGF-23, Fibroblast growth factor-23; GCS, Glasgow Coma Scale; IABP: intra-aortic balloon pump; IE, inotropic equivalent; ICU, intensive care unit; IHD, intermittent hemodialysis; KIM-1, Kidney Injury Molecule-1; LVEF, Left ventricular ejection fraction; MDRD, Modification of Diet in Renal Disease; MODS, Multiple Organ Dysfunction Syndrome; NGAL, neutrophil gelatinase-associated lipocalin; SLED, sustained low efficiency dialysis; SOFA, Sequential Organ Failure Assessment; Vit D, vitamin D. 
Area under the receiver operating characteristic (AUROC) curves were generated to evaluate biomarker performance. We use the methods of Hanley \& McNeil (PMID, 6878708) for the calculation of the Standard Error of the Area Under the Curve (AUC) and of the difference between two AUCs. A generalized additive model (GAM) (with spline), incorporating the subject-specific (longitudinal) random effects, was plotted with adjustment for other clinical parameters to assess outcome-predictive effects of candidate biomarkers in individual patients $[25,26]$.

Nonlinear effects of continuous covariates were explored with simple and multiple GAMs, which determine appropriate cut-off point(s) for discriminating candidate biomarkers, if necessary, during the stepwise variable selection procedure. The optimal cut-off value was defined as the log odd equaling zero [27].

Because of the high mortality rate among dialysis patients, competing-risk regression using the Fine and Gray model by considering the subdistribution hazard was also performed [28].

Net re-classification improvement (NRI) and integrated discrimination improvement (IDI) were used to evaluate the ability of candidate biomarkers for more accurate stratification of individuals into higher or lower risk categories (re-classification). Regarding 90-day mortality, an increase in NRI was calculated in a model containing both the AKI risk predicting score [21] and the cFGF-23 measurements, and the result was compared with the AKI risk predicting score alone. We defined $0-20 \%, 20-80 \%$, and $>80 \%$ as risk categories and re-classified patients with mortality by decision curve analysis and scatter plot (Supplemental Data file). A $p<0.05$ was considered significant.

\section{Results}

\subsection{Clinical Characteristics}

Two hundred and fifty-seven patients (mean age 65.7 years; 167 (65\%) male) with AKI who required RRT were studied. Average SOFA, APACHE II, and MODS scores were respectively 8.9, 16.3, and 5.9 .

The main causes of AKI were shock (58.4\%), sepsis (38.1\%) and contrast nephrotoxicity $(14.4 \%)$. Nine patients (3.5\%) had stage 1 AKI, 58 (22.6\%) patients had stage 2 AKI, and $190(73.9 \%)$ patients had stage 3 AKI at RRT initiation. The most frequent indication for RRT was oliguria (64.6\%), followed by azotemia ( $47.9 \%)$ and fluid overload $(43.2 \%)$ (Table 1$)$.

\subsection{Hospital and 90-Day Outcomes}

The in-hospital mortality rate, composite outcome at discharge, 90-day mortality rate and 90-day composite outcome rate were respectively $48.2 \%, 67.3 \%, 52.5 \%$, and $70.4 \%$. Table 1 shows baseline characteristics, pre-RRT and outcome parameters of patients categorized by 90 -day mortality and 90-day composite outcome, respectively. Patients who did not survive at 90 days or with a 90-day composite outcome were older, had lower urine output, higher disease severity, risk predicting scores and received higher doses of inotropic equivalents than survivals (Table 1).

Importantly, only higher plasma cFGF-23 levels enabled to differentiate patients with both 90-day mortality/composite outcomes from those without events $(p=0.001)$.

\subsection{Discriminative Power of Biomarkers for 90-Day Relevant Outcomes}

Levels of SOFA (AUROC, 0.706), AKI risk predicting score (0.677), sCr (0.619), cFGF-23 (0.687), plasma iFGF-23 (0.504), creatinine-adjusted urine NGAL (0.599), adjusted urine cFGF-23 (0.653) and adjusted urine KIM-1 (0.547) at initiation of dialysis could predict 90-day mortality. Plasma cFGF-23 demonstrated better discriminative ability than NGAL for mortality at 90 days ( $p=0.001$ by AUROC comparison) (Figure 1, $p$ at Table S1). 


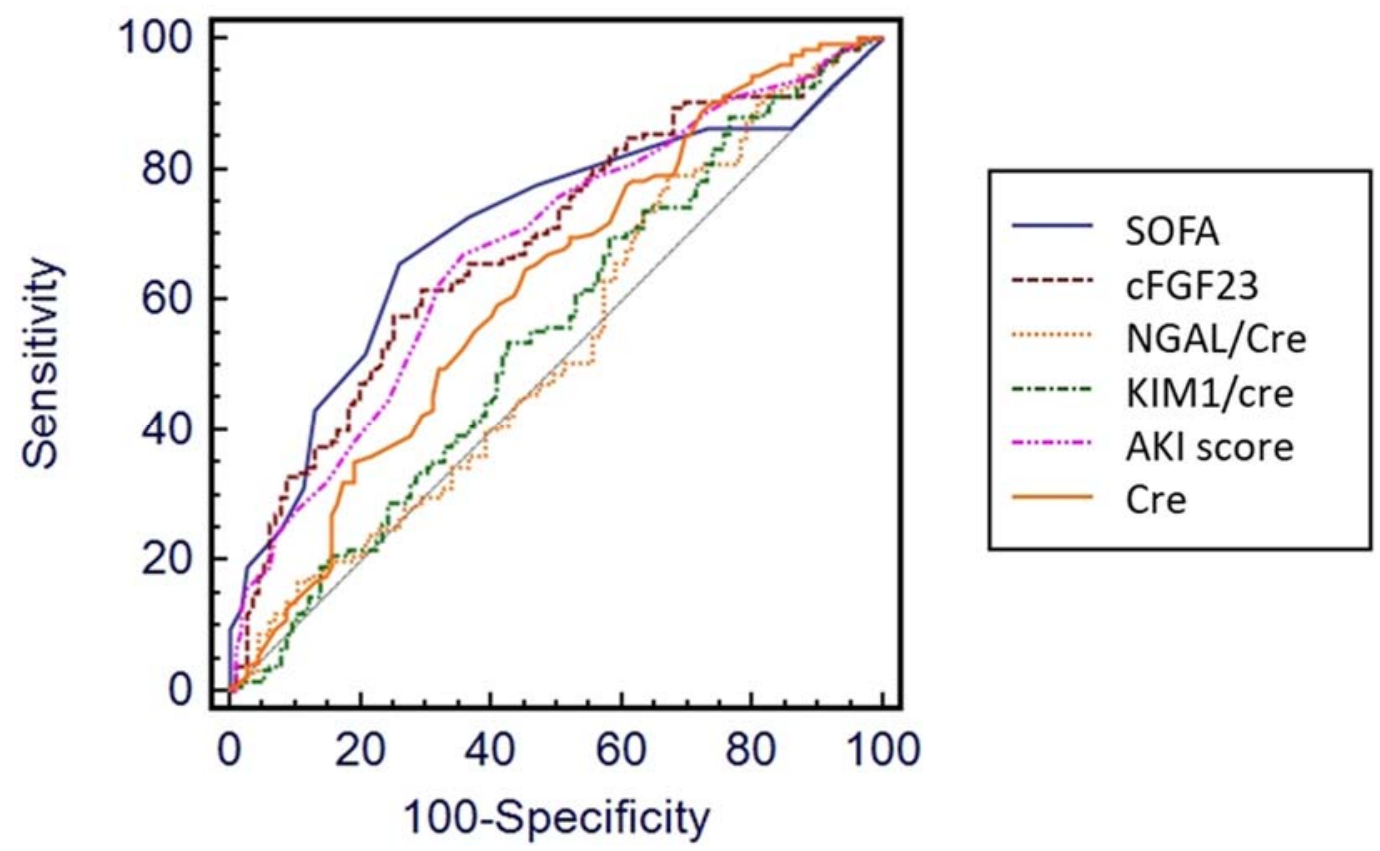

Figure 1. Comparisons of predictive powers for 90-day mortality among different variables. Note: the comparison was performed using the area under the receiver operator characteristic curves (AUROCs). Abbreviations: Cre, creatinine; cFGF-23, c-terminal fibroblast growth factor-23; KIM-1, Kidney Injury Molecule-1; NGAL, neutrophil gelatinase-associated lipocalin; SOFA, Sequential Organ Failure Assessment; KDIGO, Kidney Disease Improving Global Outcomes; AKI, acute kidney injury.

The GAM plot showed a positive correlation between increased plasma cFGF-23 levels at start of dialysis and the log of the odds of 90-day mortality and composite outcome. After adjusting all variables listed in Table 1 for nonlinear effects, plasma cFGF-23, at a cut-off value of $2050 \mathrm{RU} / \mathrm{mL}$ by the GAM model, demonstrated independently good prediction of both 90-day mortality (Figure 2A) and 90-day composite outcome (Figure 2B).

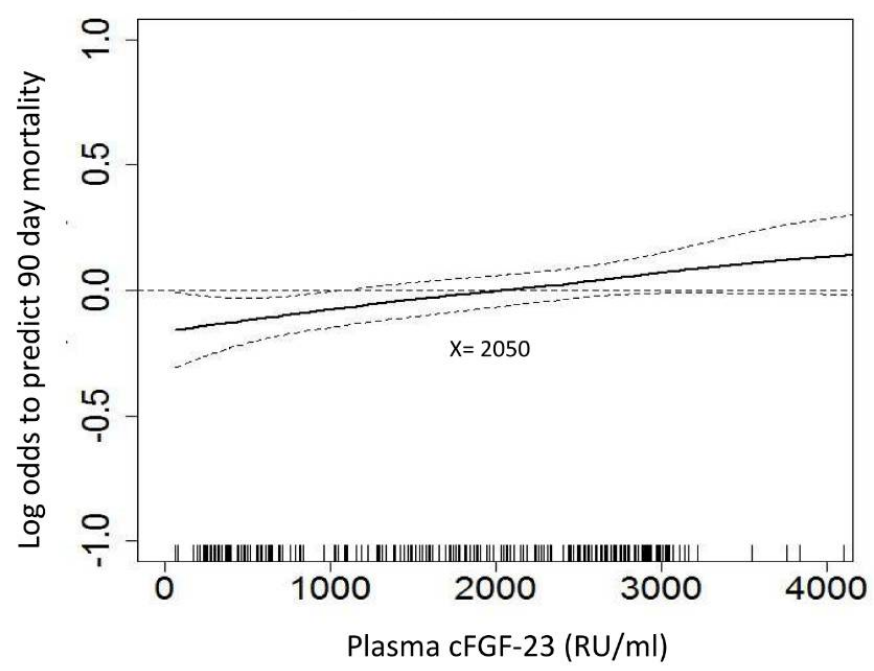

(A)

Figure 2. Cont. 


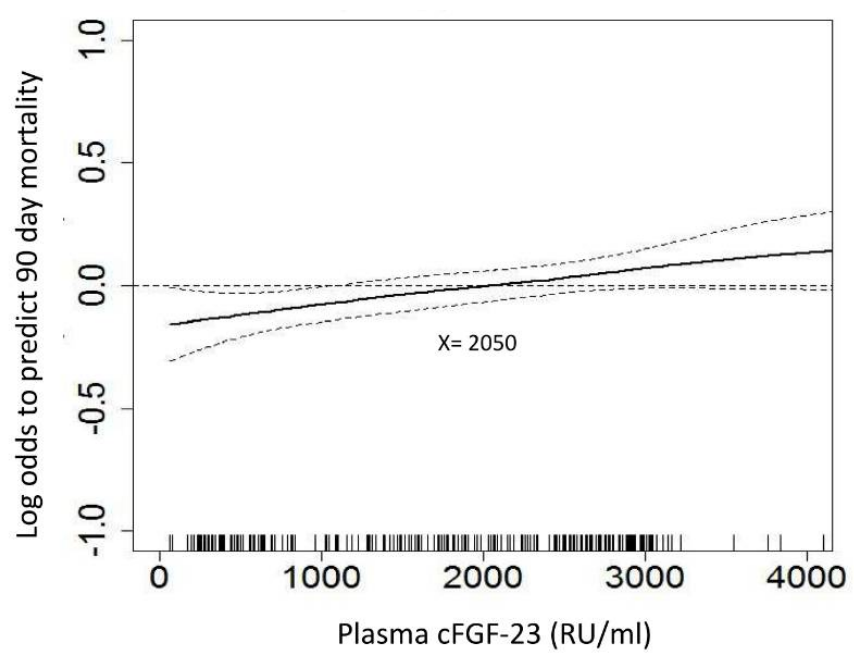

(B)

Figure 2. Generalized additive model (GAM) plot for the probability of (A) 90-day mortality, and (B) 90-day composite outcome against serum cFGF-23 levels at initiation of dialysis. Note: The GAM plot was incorporated with the subject-specific (longitudinal) random effects expressed as the logarithm of the odds (logit). The probability of outcome events was constructed with cFGF-23 levels averaging zero over the range of the data, i.e., cFGF- $23=2050 \mathrm{ng} / \mathrm{mL}$. All the relevant covariates, including characteristics, comorbidities, laboratory data, at intensive care unit (ICU) admission, etiology of acute kidney injury (AKI), indication for dialysis, dialysis modality, SOFA score, and plasma cFGF-23 at dialysis, and some of their interactions, such as interventions listed in Table 1, were put on a selected variable list to predict the outcome of interest.

\subsection{Plasma cFGF-23 and Outcome}

Using a cut-off value of $2050 \mathrm{RU} / \mathrm{mL}$, patients were divided in a "high" and a "low" cFGF-23 group. Subjects with high cFGF-23 had lower baseline sCr, but higher phosphate concentrations at dialysis initiation, higher in-hospital and 90-day mortality, lower dialysis weaning rate and higher composite outcome results (Table 2).

Table 2. Clinical characteristics of patients with high versus low plasma cFGF-23 levels.

\begin{tabular}{cccc}
\hline Serum cFGF23 Categories & Low cFGF-23 & High cFGF-23 & \multirow{2}{*}{$\boldsymbol{p}$} \\
\cline { 2 - 3 } & $(\boldsymbol{n}=\mathbf{1 1 6})$ & $\mathbf{( n = 1 4 1 )}$ & \\
\hline Patient characteristics & & & \\
\hline Age (years) & $65.8 \pm 16.0$ & $65.7 \pm 17.2$ & 0.973 \\
Gender (male) & $77(66.4 \%)$ & $90(63.8 \%)$ & 0.695 \\
Baseline creatinine (mg/dL) & $2.2 \pm 1.9$ & $1.8 \pm 1.3$ & 0.039 \\
eGFR (MDRD) (mL/min/1.73 m $\left.{ }^{2}\right)$ & $54.4 \pm 42.5$ & $56.5 \pm 39.8$ & 0.690 \\
\hline Comorbidities & & & \\
\hline Diabetes mellitus & $33(43.4 \%)$ & $82(45.3 \%)$ & 0.891 \\
Cirrhosis & $1(0.9 \%)$ & $8(5.7 \%)$ & 0.044 \\
COPD & $7(6.0 \%)$ & $8(5.7 \%)$ & 0.999 \\
CAD & $27(23.3 \%)$ & $27(19.1 \%)$ & 0.445 \\
CVA & $12(10.3 \%)$ & $12(8.5 \%)$ & 0.670 \\
\hline
\end{tabular}


Table 2. Cont.

\begin{tabular}{|c|c|c|c|}
\hline \multirow{2}{*}{ Serum cFGF23 Categories } & Low cFGF-23 & High cFGF-23 & \multirow{2}{*}{$p$} \\
\hline & $(n=116)$ & $(n=141)$ & \\
\hline Congestive heart failure & & & 0.265 \\
\hline 0 & $32(27.6 \%)$ & $35(24.8 \%)$ & \\
\hline $\mathrm{I}$ & $37(31.9 \%)$ & $63(44.7 \%)$ & \\
\hline II & $27(23.3 \%)$ & $24(17.0 \%)$ & \\
\hline III & $15(12.9 \%)$ & $16(11.3 \%)$ & \\
\hline IV & $0(0 \%)$ & $8(5.5 \%)$ & \\
\hline \multicolumn{4}{|c|}{ Laboratory data at ICU admission } \\
\hline BUN (mg/dL) & $48.3 \pm 36.1$ & $47.8 \pm 31.4$ & 0.897 \\
\hline $\mathrm{pH}$ & $7.4 \pm 0.1$ & $7.4 \pm 0.1$ & 0.354 \\
\hline $\mathrm{FiO} 2$ & $0.5 \pm 0.2$ & $0.5 \pm 0.2$ & 0.609 \\
\hline SBP & $126.1 \pm 29.2$ & $116.7 \pm 27.0$ & 0.008 \\
\hline GCS & $11.9 \pm 4.3$ & $11.9 \pm 4.2$ & 0.984 \\
\hline SOFA & $8.2 \pm 3.6$ & $9.6 \pm 3.3$ & 0.001 \\
\hline APACHE II & $15.9 \pm 6.0$ & $16.6 \pm 6.4$ & 0.405 \\
\hline MODS & $5.7 \pm 3.5$ & $6.1 \pm 3.8$ & 0.328 \\
\hline \multicolumn{4}{|l|}{ Etiology of AKI } \\
\hline Shock & $66(56.9 \%)$ & $84(59.6 \%)$ & 0.704 \\
\hline Sepsis & $40(34.5 \%)$ & $58(41.1 \%)$ & 0.303 \\
\hline Rhabdomyolysis & $7(6.0 \%)$ & $2(1.4 \%)$ & 0.083 \\
\hline Drug-induced & $2(1.7 \%)$ & $1(0.7 \%)$ & 0.591 \\
\hline Pigmentation & $5(4.3 \%)$ & $1(0.7 \%)$ & 0.094 \\
\hline Contrast & $17(14.7 \%)$ & $20(14.2 \%)$ & 0.999 \\
\hline Others & $12(10.3 \%)$ & $14(9.9 \%)$ & 0.999 \\
\hline \multicolumn{4}{|l|}{ At initiating dialysis } \\
\hline Admission to dialysis (days) & $35.5 \pm 34.1$ & $42.6 \pm 45.4$ & 0.163 \\
\hline Mechanical ventilation & $78(67.2 \%)$ & $107(75.9 \%)$ & 0.128 \\
\hline Emergency Surgery & $45(38.8 \%)$ & $55(39.0 \%)$ & 0.999 \\
\hline IABP & $13(11.2 \%)$ & $14(9.9 \%)$ & 0.839 \\
\hline Urine output (mL/24 h) & $650.9 \pm 642.9$ & $542.9 \pm 892.8$ & 0.277 \\
\hline AKI risk prediction score & $21.5 \pm 6.7$ & $23.5 \pm 6.9$ & 0.021 \\
\hline Body weight $(\mathrm{kg})$ & $67.2 \pm 15.4$ & $66.5 \pm 13.3$ & 0.728 \\
\hline IE & $7.14 \pm 11.5$ & $9.1 \pm 17.4$ & 0.310 \\
\hline SOFA & $10.6 \pm 4.3$ & $11.2 \pm 3.6$ & 0.218 \\
\hline APACHE II & $17.8 \pm 6.7$ & $11.8 \pm 6.2$ & 0.980 \\
\hline MODS & $7.8 \pm 4.4$ & $8.4 \pm 3.8$ & 0.222 \\
\hline Phosphate, mg/dL & $4.1 \pm 1.7$ & $4.9 \pm 1.7$ & 0.021 \\
\hline $25 \mathrm{OH}$ Vit D, ng/mL & $11.0 \pm 5.8$ & $12.5 \pm 5.6$ & 0.617 \\
\hline 1,25 diOH Vit D, pg/mL & $29.7 \pm 6.9$ & $25.0 \pm 5.5$ & 0.149 \\
\hline \multicolumn{4}{|l|}{ Kidney function marker } \\
\hline BUN (mg/dL) & $81.2 \pm 45.8$ & $83.4 \pm 48.4$ & 0.714 \\
\hline Creatinine (mg/dL) & $4.2 \pm 2.4$ & $4.1 \pm 2.3$ & 0.677 \\
\hline Urine KIM1 (ng/mL) & $5.9 \pm 5.9$ & $6.1 \pm 5.7$ & 0.800 \\
\hline Urine KIM1/Cre & $0.13 \pm 0.18$ & $0.14 \pm 0.14$ & 0.715 \\
\hline Urine NGAL (ng/mL) & $196.5 \pm 86.1$ & $198.2 \pm 85.0$ & 0.916 \\
\hline Urine NGAL/Cre & 7.012 .9 & $6.8 \pm 9.4$ & 0.877 \\
\hline Urine cFGF-23/Cre & $523.4 \pm 747.2$ & $1173.3 \pm 1077.6$ & $<0.001$ \\
\hline Plasma iFGF-23 (pg/mL) & $257.6 \pm 243.0$ & $325.50 \pm 542.3$ & 0.536 \\
\hline
\end{tabular}


Table 2. Cont.

\begin{tabular}{cccc}
\hline Serum cFGF23 Categories & Low cFGF-23 & High cFGF-23 & \multirow{2}{*}{$\boldsymbol{p}$} \\
\cline { 2 - 3 } & $(\boldsymbol{n}=\mathbf{1 1 6})$ & $\mathbf{( n = 1 4 1 )}$ & \\
\hline Indication for dialysis & & & \\
\hline Azotemia & $56(48.3 \%)$ & $67(47.5 \%)$ & 0.999 \\
Fluid overload & $48(41.4 \%)$ & $63(44.7 \%)$ & 0.615 \\
Electrolyte disorders & $7(60 \%)$ & $11(7.8 \%)$ & 0.631 \\
Metabolic acidosis & $22(19.0 \%)$ & $24(17.0 \%)$ & 0.745 \\
Oliguria & $73(62.9 \%)$ & $93(66.0 \%)$ & 0.694 \\
Uremic complication & $7(6.0 \%)$ & $5(3.5 \%)$ & 0.386 \\
\hline Dialysis modality & & & 0.011 \\
\hline CVVH & $44(37.9 \%)$ & $31(22.0 \%)$ & \\
IHD & $21(18.1 \%)$ & $41(29.1 \%)$ & \\
SLED & $51(44.0 \%)$ & $69(48.9 \%)$ & \\
\hline Outcomes of interest & & & \\
\hline Hospital length of stay (days) & $49.0 \pm 43.0$ & $59.4 \pm 55.5$ & 0.101 \\
Duration of hospital dialysis (days) & $39.9 \pm 34.4$ & $39.0 \pm 45.5$ & 0.862 \\
Hospital mortality & $42(36.2 \%)$ & $82(58.2 \%)$ & $<0.001$ \\
Composite outcome at discharge & $69(59.5 \%)$ & $104(73.8 \%)$ & $<0.001$ \\
90-day mortality & $45(38.8 \%)$ & $90(63.8 \%)$ & $<0.001$ \\
90-day weaning from dialysis & $47(40.5 \%)$ & $29(20.6 \%)$ & $<0.001$ \\
90-day composite outcome & $69(59.5 \%)$ & $112(79.4 \%)$ & $<0.001$ \\
\hline
\end{tabular}

Abbreviations: AKI, acute kidney injury; APACHE; Acute Physiology and Chronic Health Evaluation, BMI, body mass index; $\mathrm{CABG}$, coronary artery bypass graft; $\mathrm{Cre}$, creatinine; $\mathrm{BUN}$, blood urea nitrogen; $\mathrm{COPD}$, chronic obstructive pulmonary disease; $\mathrm{CPB}$, cardiopulmonary bypass; Cre, creatinine; $\mathrm{CVA}$, cerebrovascular accident; CVVH, continuous venovenous hemofiltration; eGFR, estimated glomerular filtration rate; FGF-23, Fibroblast growth factor-23; GCS, Glasgow Coma Scale; IABP: intra-aortic balloon pump; IE, inotropic equivalent; ICU, intensive care unit; IHD, intermittent hemodialysis; KIM-1, Kidney Injury Molecule-1; LVEF, Left ventricular ejection fraction; MDRD, Modification of Diet in Renal Disease; MODS, Multiple Organ Dysfunction Syndrome; NGAL, neutrophil gelatinase-associated lipocalin; SLED, sustained low efficiency dialysis; SOFA, Sequential Organ Failure Assessment; Vit D, vitamin D.

A high cFGF-23 level represented an independent risk factor for in-hospital mortality (OR, 1.80, $p=0.049)$, composite outcome at discharge ( $\mathrm{OR}, 1.80,95 \% \mathrm{CI}=1.01-3.24 ; p=0.043), 90$-day mortality $(\mathrm{OR}, 2.19,95 \% \mathrm{CI}=1.20-4.00 ; p=0.011)$, and 90-day composite outcome $(\mathrm{OR}, 2.39,95 \% \mathrm{CI}=1.31-4.35$; $p=0.005$ ) after adjusting for age, gender, baseline eGFR, and factor interaction with cFGF-23 and SOFA score. Importantly, no interaction was observed between the cFGF-23 level and underlying diabetes mellitus, baseline eGFR, age, and AKI risk predicting score at dialysis initiation (all $p>0.05$ ) (Table 3 ).

Table 3. Logistic regression model for mortality and composite outcomes at hospital discharge and 90 days after discharge. Significant risks were shown.

\begin{tabular}{ccccccc}
\hline \multirow{2}{*}{ Independent Variables } & \multicolumn{3}{c}{ Hospital Mortality } & \multicolumn{3}{c}{ Composite Outcome at Discharge } \\
\cline { 2 - 7 } & OR & $\mathbf{9 5 \%}$ CI & $p$ & OR & $\mathbf{9 5 \% ~ C I ~}$ & $p$ \\
\hline Age (per year) & 1.03 & $1.01-1.04$ & 0.007 & 1.03 & $1.01-1.04$ & 0.004 \\
SOFA (per score) & 1.26 & $1.15-1.39$ & $<0.001$ & 1.12 & $1.03-1.22$ & 0.011 \\
High cFGF-23 & 1.80 & $1.01-3.24$ & 0.043 & 1.80 & $1.01-3.19$ & 0.045 \\
\hline \multicolumn{3}{c}{ 90-Day Mortality } & 90-Day Composite Outcome \\
\hline Age (per year) & 1.03 & $1.01-1.05$ & 0.001 & 1.03 & $1.01-1.05$ & 0.001 \\
SOFA (per score) & 1.30 & $1.17-1.44$ & 0.037 & 1.17 & $1.07-1.27$ & $<0.001$ \\
High cFGF-23 & 2.19 & $1.20-4.00$ & 0.011 & 2.39 & $1.31-4.35$ & 0.005 \\
\hline
\end{tabular}

Abbreviations: cFGF-23, c-terminal fibroblast growth factor-23; CI, confidence interval; HR, hazard ratio; SOFA, Sequential Organ Failure Assessment. All the univariate significant and non-significant relevant covariates, including age, sex, baseline comorbidities, indication for dialysis, etiology of AKI, kidney function profile (e.g., baseline eGFR and candidate biomarkers), cFGF-23 and SOFA score at dialysis initiation, dialysis modality, and some of their interactions were put on the variable lists to be selected (Table 1). 
Cox proportional hazard regression analysis revealed that patients undergoing RRT who displayed high cFGF-23 levels had a higher 90-day mortality during the follow-up period with an adjusted HR of $1.76(95 \% \mathrm{CI}, 1.22-2.53 ; p=0.020)$ as compared with patients with lower cFGF-23 values (Figure 3A). There was no interaction of the baseline comorbidities with high cFGF-23 to predict 90-day composite outcome. (Table S2) Taking mortality as a competing risk factor for dialysis, high cFGF-23 levels also predicted less weaning from dialysis in surviving patients (HR, 0.62, $p=0.032$ ) (Figure 3B).

\section{Cox proportional hazards regression}

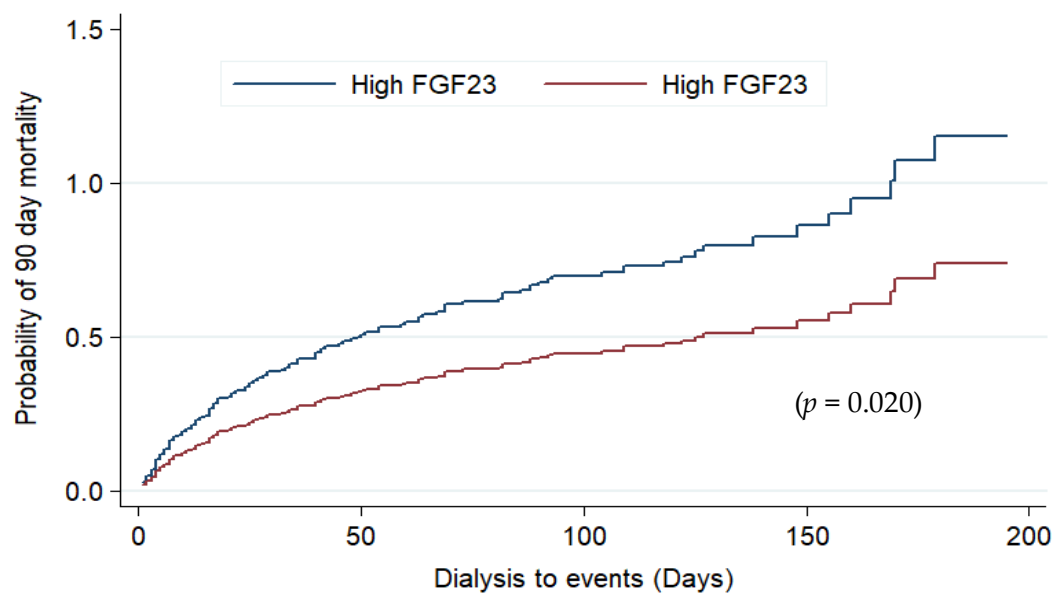

(A)

\section{Competing-risks regression}

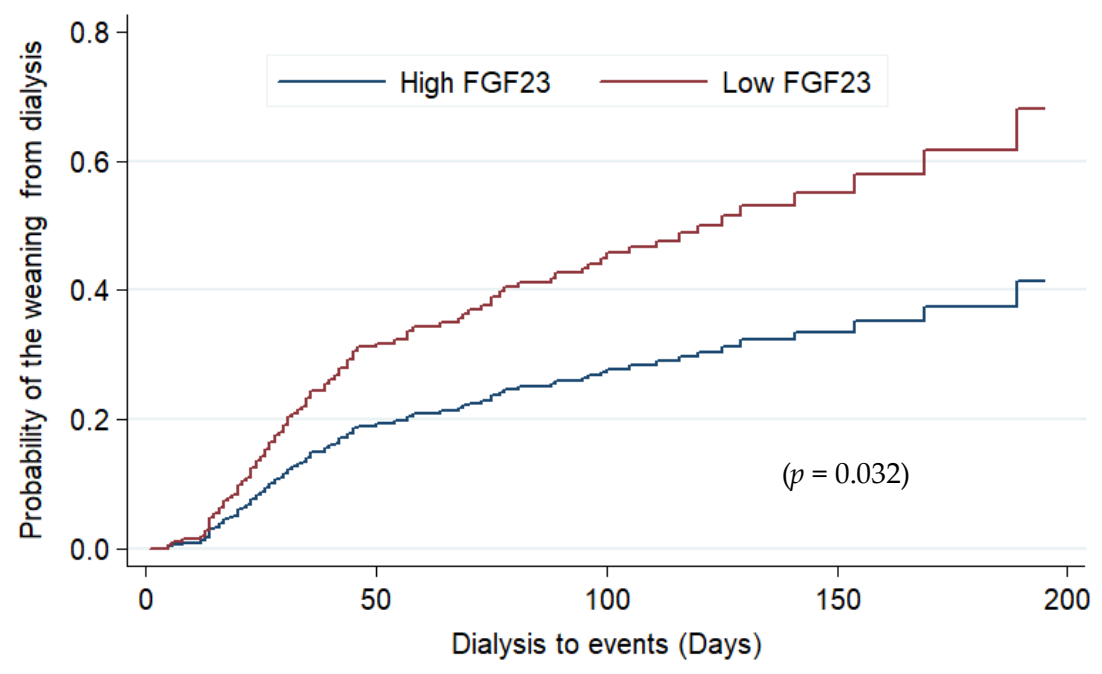

(B)

Figure 3. Cox proportional hazard plots stratified by serum cFGF-23 level for assessing probability of 90-day mortality (A) and the weaning from dialysis (B) by competing analysis and with mortality as a risk factor. Abbreviations: cFGF-23, c-terminal fibroblast growth factor-23; Using a cut-off value of $2050 \mathrm{RU} / \mathrm{mL}$ of cFGF-23 at initializing dialysis, patients were divided in a "high" and a "low" cFGF-23 group; all the relevant covariates, including characteristics, comorbidities, laboratory data, at ICU admission, etiology of AKI, indication for dialysis, dialysis modality, SOFA score, and plasma cFGF-23 at dialysis, and some of their interactions, such as interventions listed in Table 1, were put on a selected variable list to predict the outcome of interest. 
The relationship of cFGF-23 with these variables was also underscored by a GAM analysis adjusted for SOFA score, gender, and age, which showed that cFGF-23 levels correlated with iFGF-23 $(p=0.013)$ and SOFA score $(p<0.001)$, but not with $\mathrm{sCr}(p=0.116)$, phosphate $(p=0.591), 25$-hydroxy Vit $\mathrm{D}(p=0.485)$ and 1,25 dihydroxy vit $\mathrm{D}(p=0.638)$ concentrations at initiating RRT (Figure S1).

\subsection{Addition of cFGF-23 to AKI Risk Predicting Score at Start of Dialysis}

Adding cFGF-23 to the AKI risk predicting score at dialysis initiation significantly increased risk stratification (total NRI $=0.148 ; 95 \% \mathrm{CI}=0.057-0.239 ; p=0.002$ ) for detection of 90 -day mortality. This effect was primarily determined by death (NRI event $=0.068,95 \% \mathrm{CI}=0.043-0.087 ; p=0.025$ ) and survival (NRI event $=0.069 ; 95 \% \mathrm{CI}=0.039-0.097 ; p=0.029)$. Similarly, the total IDI was significant. $(0.051,95 \% \mathrm{CI}=0.024-0.079 ; p<0.001)$ (Figures S2 and S3).

\section{Discussion}

At initializing dialysis, the discriminative power of AKI biomarkers for 90-day mortality is fair. At dialysis initiation, the discrimination of cFGF-23 is better than NGAL, KIM-1, iFGF-23 and creatinine predicting patients' outcome. With mortality as competing risk, higher cFGF-23 levels also predicted lesser kidney recovery in survivors. More importantly, cFGF-23 had better predictive power than creatinine-adjusted urine NGAL and its integration into the AKI risk predicting score significantly enhanced the accuracy of risk stratification. At a cut-off level above $2050 \mathrm{RU} / \mathrm{mL}$, cFGF-23 could predict of AKI mortality after adjusting for different clinical and disease severity parameters. Thus, cFGF-23 could be used as an early determinant of prognosis in ICU patients subjected at initializing RRT and also as an early determinant of the timing of dialysis initiation.

An increasing body of evidence has shown that cFGF-23 levels are increased in patients with AKI [11,14,29-32]. No significant interaction was observed between cFGF-23 and baseline CKD, sepsis grading in predicting mortality. The SOFA score was independently associated with increased cFGF-23 levels, which underpins the potential use of cFGF-23 in a critical care setting. We dare suggest that a higher plasma cFGF-23 not only corresponds with more severe AKI, but also reflects a higher degree of systemic inflammation.

Several mechanisms may explain increased FGF-23 levels in AKI: (1) increased production by osteocytes and possibly osteoblasts, that escapes regulation by parathyroid hormone, vitamin $\mathrm{D}$ signaling, and dietary phosphate restriction [33,34]; (2) increased ectopic production of FGF-23 by damaged renal tubules [33,35]; (3) tubular dysfunction resulting in FGF-23 resistance [36]; (4) and decreased clearance of circulating FGF-23 [14]. Whilst circulating FGF-23 levels rise rapidly during AKI [14] and a causal role for FGF-23 in the pathogenesis of left ventricular hypertrophy has previously been unveiled, suggesting that chronically elevated FGF-23 levels contribute directly to cardiac mortality in patients with CKD [37].

The ideal circumstances for whether and when to start RRT remain unclear [4]. We found significantly elevated cFGF-23 levels at the start of dialysis in non-survivors, whilst other structural and functional renal biomarkers failed to discriminate. Elevated plasma cFGF-23 was related to the degree of organ failure at initializing RRT [33]. In fact, high cFGF-23 concentrations predicted worse outcome equally well as the SOFA score in critically ill patients with advanced AKI [38]. Moreover, in patients without AKI, plasma cFGF-23 levels were significantly higher in the more severely ill patients [14]. This underscores that high cFGF-23 levels are correlated with increased systemic inflammation and/or stress secondary to illness or major surgery [33]. Although both serum and urine cFGF-23 could predict AKI mortality after ICU admission [12], many patients were oliguric at initializing dialysis, that will highlight the role of serum cFGF-23. In surviving patients, high cFGF-23 levels also predicted a lesser possibility for RRT withdrawal. Early prediction of renal recovery is likely to be helpful with regard to post-discharge care after critical illness and subsequent progression to CKD and ESRD.

Taken together, the ability of cFGF-23 to predict adverse outcomes might be related more to the systemic inflammatory status than to tubular damage. Based on our findings, a prognostic model can 
be constructed that allows to predict individual mortality risk as well as potential kidney recovery in surviving patients before starting RRT. The addition of cFGF-23 to a clinical AKI risk predicting score resulted in greater discrimination, and enhanced the ability to anticipate a higher number of subsequent deaths. Given the lack of appropriate or reliable biomarkers in patients receiving RRT, plasma cFGF-23 tentatively may serve as a novel outcome-specific marker in critical care nephrology. In patients with augmented plasma cFGF-23 concentration to arrive $2050 \mathrm{RU} / \mathrm{mL}$, the clinician should evaluate the traditional AKI risk score or parameters to decide commencing dialysis.

Whether the cFGF-23 assay provides comparable sensitivity to that for iFGF-23 in patients with different stages of AKI or illness severity is still debated [13]. Although measurements obtained with iFGF-23 and cFGF-23 assays reflect the same circulating moiety, it has been suggested that the levels of iFGF23 also increased in patients who developed severe AKI, but the magnitude was lower than cFGF23 [13]. This is also supported by the present study showing that a plasma cFGF-23 concentration exceeding $2050 \mathrm{RU} / \mathrm{mL}$ at initializing RRT was significantly associated with worse patient outcome at a higher discriminative power than iFGF-23. The levels of adjusted urine cFGF23 also increased in patients who did not survive, but the magnitude was lower than serum cFGF23.

Several limitations of our study must be highlighted. Our cFGF-23 cutoff value was somewhat higher than that in other AKI studies [11-15], probably because most patients already had advanced AKI when admitted to the ICU. Furthermore, the predicting power of CFGF-23 in patients without AKI but with high inflammation status needs further validation. Finally, the exact mechanism underlying increased cFGF-23 concentrations in AKI patients as well as possible other intrinsic biological effects of cFGF-23 in this particular population remain to be explored. As previously studies few biomarkers were ever validated and they could only modestly predictive of renal recovery [8]; we do acknowledge also that the AUCs of cFGF-23 were relatively modest in AKI-D patients with critical status, however adding cFGF-23 to a parsimonious model augmented prediction of mortality and kidney recovery.

\section{Conclusions}

At initializing dialysis, the discriminative power of AKI biomarkers for 90-day mortality is fair. Our study showed that cFGF-23, measured at initiation of RRT in critical patients with AKI, may be a novel and distinct marker for predicting 90-day mortality after discharge and less weaning from RRT in survivors. Its predictive discrimination was superior to other established biomarkers of kidney injury, in particular creatinine, NGAL and Kim-1. Adding cFGF-23 to the traditional AKI risk predicting score may allow better risk stratification and enhance prognostic power. cFGF-23 could further be used as a surrogate marker to decide the best timing to initiate RRT.

Supplementary Materials: The following are available online at http:/ www.mdpi.com/2077-0383/7/8/202/s1, Figure S1: Scatter plots with an adjusted spline of cFGF23 with (A) iFGF23 $(p=0.013)$, (B) phosphate $(p=0.591)$ (C) creatinine $(p=0.116)(\mathrm{D}) 25 \mathrm{OH}$ Vitamin $\mathrm{D}(p=0.485)$ and (E) $1,25 \mathrm{OH}$, Vitamin $\mathrm{D}(p=0.638)$ (F) KDIGO-AKI score $(p=0.820)(\mathrm{G})$ SOFA $(p<0.001)$ at initiation of dialysis, Figure S2: Decision curve analysis (DCA) plot to assess 90 day mortality using cFGF-23 in addition to AKI risk prediction score, Figure S3: The correlation of AKI risk predicting score and AKI risk predicting score with cFGF-23 predicting 90 day mortality, Table S1: $p$ value comparison of the receiver operating characteristic (ROC) curve for discriminative ability, Table S2: Interaction of baseline co-morbidity with high cFGF-23 to predict 90-day composite outcome.

Author Contributions: V.-C.W., C.-C.S., S.-C.J.C., H.-H.L. and T.-S.C. conceived the review topic, analysis and interpretation and wrote the manuscript. H.D.S., N.-H.C., C.-H.W. and P.M.H. revised and approved the final version of the manuscript.

Funding: This study was supported by Taiwan National Science Council (grants NSC 102-2314-B-002-140-MY2, 104-2314-B-002-125-MY3, 106-2314-B-002-166-MY3, MOST 106-2321-B-182-002) and NTUH 106-FTN20, 106-P02, UN106-014, 107-T02.

Acknowledgments: We also express our sincere gratitude to all staff of the Taiwan Clinical Trial Consortium, TCTC, and extracorporeal membrane oxygenation team in NTUH. All authors read and approved the final version of the manuscript.

Conflicts of Interest: The authors declare no conflict of interest. 


\section{Abbreviations}

$\begin{array}{ll}\text { AKI } & \text { acute kidney injury } \\ \text { APACHE II } & \text { Acute Physiology and Chronic Health Evaluation II } \\ \text { AUROC } & \text { area under the receiver operator characteristic curve } \\ \text { CKD } & \text { chronic kidney disease } \\ \text { ESRD } & \text { end-stage renal disease } \\ \text { ICU } & \text { intensive care unit } \\ \text { KIM-1 } & \text { Kidney Injury Molecule-1 } \\ \text { MODS } & \text { Multiple Organ Dysfunction Score } \\ \text { NGAL } & \text { neutrophil gelatinase-associated lipocalin } \\ \text { RRT } & \text { renal replacement therapy } \\ \text { RU } & \text { relative units } \\ \text { SCr } & \text { serum creatinine } \\ \text { SOFA } & \text { Sequential Organ Failure Assessment }\end{array}$

\section{References}

1. Shiao, C.C.; Wu, P.C.; Huang, T.M.; Lai, T.S.; Yang, W.S.; Wu, C.H.; Lai, C.F.; Wu, V.C.; Chu, T.S.; Wu, K.D.; et al. Long-term remote organ consequences following acute kidney injury. Crit. Care 2015, 19, 438. [CrossRef] [PubMed]

2. Wu, V.C.; Shiao, C.C.; Chang, C.H.; Huang, T.M.; Lai, C.F.; Lin, M.C.; Chiang, W.C.; Chu, T.S.; Wu, K.D.; Ko, W.J.; et al. Long-term outcomes after dialysis-requiring acute kidney injury. Biomed. Res. Int. 2014, 2014, 365186. [CrossRef] [PubMed]

3. Wald, R.; Bagshaw, S.M. The timing of renal replacement therapy initiation in acute kidney injury: Is earlier truly better? Crit. Care Med. 2014, 42, 1933-1934. [CrossRef] [PubMed]

4. Klein, S.J.; Brandtner, A.K.; Lehner, G.F.; Ulmer, H.; Bagshaw, S.M.; Wiedermann, C.J.; Joannidis, M. Biomarkers for prediction of renal replacement therapy in acute kidney injury: A systematic review and meta-analysis. Intensive Care Med. 2018, 44, 323-336. [CrossRef] [PubMed]

5. Kumpers, P.; Hafer, C.; Lukasz, A.; Lichtinghagen, R.; Brand, K.; Fliser, D.; Faulhaber-Walter, R.; Kielstein, J.T. Serum neutrophil gelatinase-associated lipocalin at inception of renal replacement therapy predicts survival in critically ill patients with acute kidney injury. Crit. Care 2010, 14, R9. [CrossRef] [PubMed]

6. Zarbock, A.; Kellum, J.A.; Schmidt, C.; Van Aken, H.; Wempe, C.; Pavenstadt, H.; Boanta, A.; Gerss, J.; Meersch, M. Effect of Early vs Delayed Initiation of Renal Replacement Therapy on Mortality in Critically Ill Patients With Acute Kidney Injury: The Elain Randomized Clinical Trial. JAMA 2016, 315, 2190-2199. [CrossRef] [PubMed]

7. Lin, C.Y.; Chang, C.H.; Fan, P.C.; Tian, Y.C.; Chang, M.Y.; Jenq, C.C.; Hung, C.C.; Fang, J.T.; Yang, C.W.; Chen, Y.C. Serum interleukin-18 at commencement of renal replacement therapy predicts short-term prognosis in critically ill patients with acute kidney injury. PLoS ONE 2013, 8, e66028. [CrossRef] [PubMed]

8. Pike, F.; Murugan, R.; Keener, C.; Palevsky, P.M.; Vijayan, A.; Unruh, M.; Finkel, K.; Wen, X.; Kellum, J.A. Biomarker Enhanced Risk Prediction for Adverse Outcomes in Critically Ill Patients Receiving RRT. Clin. J. Am. Soc. Nephrol. 2015, 10, 1332-1339. [CrossRef] [PubMed]

9. Consortium, A. Autosomal dominant hypophosphataemic rickets is associated with mutations in FGF23. Nat. Genet. 2000, 26, 345-348.

10. Berndt, T.; Kumar, R. Phosphatonins and the regulation of phosphate homeostasis. Annu. Rev. Physiol. 2007, 69, 341-359. [CrossRef] [PubMed]

11. Ali, F.N.; Hassinger, A.; Price, H.; Langman, C.B. Preoperative plasma FGF23 levels predict acute kidney injury in children: Results of a pilot study. Pediatr. Nephrol. 2013, 28, 959-962. [CrossRef] [PubMed]

12. Leaf, D.E.; Jacob, K.A.; Srivastava, A.; Chen, M.E.; Christov, M.; Juppner, H.; Sabbisetti, V.S.; Martin, A.; Wolf, M.; Waikar, S.S. Fibroblast Growth Factor 23 Levels Associate with AKI and Death in Critical Illness. J. Am. Soc. Nephrol. 2017, 28, 1877-1885. [CrossRef] [PubMed]

13. Leaf, D.E.; Christov, M.; Juppner, H.; Siew, E.; Ikizler, T.A.; Bian, A.; Chen, G.; Sabbisetti, V.S.; Bonventre, J.V.; Cai, X.; et al. Fibroblast growth factor 23 levels are elevated and associated with severe acute kidney injury and death following cardiac surgery. Kidney Int. 2016, 89, 939-948. [CrossRef] [PubMed] 
14. Christov, M.; Waikar, S.S.; Pereira, R.C.; Havasi, A.; Leaf, D.E.; Goltzman, D.; Pajevic, P.D.; Wolf, M.; Juppner, H. Plasma FGF23 levels increase rapidly after acute kidney injury. Kidney Int. 2013, 84, 776-785. [CrossRef] [PubMed]

15. Donate-Correa, J.; de Fuentes, M.M.; Mora-Fernandez, C.; Navarro-Gonzalez, J.F. Pathophysiological implications of fibroblast growth factor-23 and Klotho and their potential role as clinical biomarkers. Clin. Chem. 2014, 60, 933-940. [CrossRef] [PubMed]

16. Wu, V.C.; Ko, W.J.; Chang, H.W.; Chen, Y.S.; Chen, Y.W.; Chen, Y.M.; Hu, F.C.; Lin, Y.H.; Tsai, P.R.; Wu, K.D. Early renal replacement therapy in patients with postoperative acute liver failure associated with acute renal failure: Effect on postoperative outcomes. J. Am. Coll. Surg. 2007, 205, 266-276. [CrossRef] [PubMed]

17. Wu, V.C.; Ko, W.J.; Chang, H.W.; Chen, Y.W.; Lin, Y.F.; Shiao, C.C.; Chen, Y.M.; Chen, Y.S.; Tsai, P.R.; $\mathrm{Hu}$, F.C.; et al. Risk factors of early redialysis after weaning from postoperative acute renal replacement therapy. Intensive Care Med. 2008, 34, 101-108. [CrossRef] [PubMed]

18. Shiao, C.C.; Wu, V.C.; Li, W.Y.; Lin, Y.F.; Hu, F.C.; Young, G.H.; Kuo, C.C.; Kao, T.W.; Huang, D.M.; Chen, Y.M.; et al. Late initiation of renal replacement therapy is associated with worse outcomes in acute kidney injury after major abdominal surgery. Crit. Care 2009, 13, R171. [CrossRef] [PubMed]

19. Wu, V.C.; Wang, C.H.; Wang, W.J.; Lin, Y.F.; Hu, F.C.; Chen, Y.W.; Chen, Y.S.; Wu, M.S.; Lin, Y.H.; Kuo, C.C.; et al. Sustained low-efficiency dialysis versus continuous veno-venous hemofiltration for postsurgical acute renal failure. Am. J. Surg. 2010, 199, 466-476. [CrossRef] [PubMed]

20. Huang, T.M.; Wu, V.C.; Young, G.H.; Lin, Y.F.; Shiao, C.C.; Wu, P.C.; Li, W.Y.; Yu, H.Y.; Hu, F.C.; Lin, J.W.; et al. Preoperative proteinuria predicts adverse renal outcomes after coronary artery bypass grafting. J. Am. Soc. Nephrol. 2011, 22, 156-163. [CrossRef] [PubMed]

21. Demirjian, S.; Chertow, G.M.; Zhang, J.H.; O'Connor, T.Z.; Vitale, J.; Paganini, E.P.; Palevsky, P.M. Network VNARFT. Model to predict mortality in critically ill adults with acute kidney injury. Clin. J. Am. Soc. Nephrol. 2011, 6, 2114-2120. [CrossRef] [PubMed]

22. Wu, V.C.; Huang, T.M.; Lai, C.F.; Shiao, C.C.; Lin, Y.F.; Chu, T.S.; Wu, P.C.; Chao, C.T.; Wang, J.Y.; Kao, T.W.; et al. Acute-on-chronic kidney injury at hospital discharge is associated with long-term dialysis and mortality. Kidney Int. 2011, 80, 1222-1230. [CrossRef] [PubMed]

23. Lin, Y.F.; Ko, W.J.; Wu, V.C.; Chen, Y.S.; Chen, Y.M.; Hu, F.C.; Shiao, C.C.; Wu, M.S.; Chen, Y.W.; Li, W.Y.; et al. A modified sequential organ failure assessment score to predict hospital mortality of postoperative acute renal failure patients requiring renal replacement therapy. Blood Purif. 2008, 26, 547-554. [CrossRef] [PubMed]

24. Shiao, C.C.; Ko, W.J.; Wu, V.C.; Huang, T.M.; Lai, C.F.; Lin, Y.F.; Chao, C.T.; Chu, T.S.; Tsai, H.B.; Wu, P.C.; et al. U-curve association between timing of renal replacement therapy initiation and in-hospital mortality in postoperative acute kidney injury. PLoS ONE 2012, 7, e42952. [CrossRef] [PubMed]

25. Wu, V.C.; Lo, S.C.; Chen, Y.L.; Huang, P.H.; Tsai, C.T.; Liang, C.J.; Kuo, C.C.; Kuo, Y.S.; Lee, B.C.; Wu, E.L.; et al. Endothelial progenitor cells in primary aldosteronism: A biomarker of severity for aldosterone vasculopathy and prognosis. J. Clin. Endocrinol. Metab. 2011, 96, 3175-3183. [CrossRef] [PubMed]

26. Wu, V.C.; Lai, C.F.; Shiao, C.C.; Lin, Y.F.; Wu, P.C.; Chao, C.T.; Hu, F.C.; Huang, T.M.; Yeh, Y.C.; Tsai, I.J.; et al. Effect of diuretic use on 30-day postdialysis mortality in critically ill patients receiving acute dialysis. PLoS ONE 2012, 7, e30836. [CrossRef] [PubMed]

27. Hin, L.Y.; Lau, T.K.; Rogers, M.S.; Chang, A.M. Dichotomization of continuous measurements using generalized additive modelling-Application in predicting intrapartum caesarean delivery. Stat. Med. 1999, 18, 1101-1110. [CrossRef]

28. Wu, V.C.; Chang, C.H.; Wang, C.Y.; Lin, Y.H.; Kao, T.W.; Lin, P.C.; Chu, T.S.; Chang, Y.S.; Chen, L.; Wu, K.D.; et al. Risk of Fracture in Primary Aldosteronism: A Population-Based Cohort Study. J. Bone Miner. Res. 2017, 32, 743-752. [CrossRef] [PubMed]

29. Zhang, M.; Hsu, R.; Hsu, C.Y.; Kordesch, K.; Nicasio, E.; Cortez, A.; McAlpine, I.; Brady, S.; Zhuo, H.; Kangelaris, K.N.; et al. FGF-23 and PTH levels in patients with acute kidney injury: A cross-sectional case series study. Ann. Intensive Care. 2011, 1, 21. [CrossRef] [PubMed]

30. Leaf, D.E.; Wolf, M.; Waikar, S.S.; Chase, H.; Christov, M.; Cremers, S.; Stern, L. FGF-23 levels in patients with AKI and risk of adverse outcomes. Clin. J. Am. Soc. Nephrol. 2012, 7, 1217-1223. [CrossRef] [PubMed] 
31. Leaf, D.E.; Waikar, S.S.; Wolf, M.; Cremers, S.; Bhan, I.; Stern, L. Dysregulated mineral metabolism in patients with acute kidney injury and risk of adverse outcomes. Clin. Endocrinol. 2013, 79, 491-498. [CrossRef] [PubMed]

32. Brown, J.R.; Katz, R.; Ix, J.H.; de Boer, I.H.; Siscovick, D.S.; Grams, M.E.; Shlipak, M.; Sarnak, M.J. Fibroblast growth factor-23 and the long-term risk of hospital-associated AKI among community-dwelling older individuals. Clin. J. Am. Soc. Nephrol. 2014, 9, 239-246. [CrossRef] [PubMed]

33. Neyra, J.A.; Moe, O.W.; Hu, M.C. Fibroblast growth factor 23 and acute kidney injury. Pediatr. Nephrol. 2015, 30, 1909-1918. [CrossRef] [PubMed]

34. Shimada, T.; Muto, T.; Urakawa, I.; Yoneya, T.; Yamazaki, Y.; Okawa, K.; Takeuchi, Y.; Fujita, T.; Fukumoto, S.; Yamashita, T. Mutant FGF-23 responsible for autosomal dominant hypophosphatemic rickets is resistant to proteolytic cleavage and causes hypophosphatemia in vivo. Endocrinology 2002, 143, 3179-3182. [CrossRef] [PubMed]

35. Spichtig, D.; Zhang, H.; Mohebbi, N.; Pavik, I.; Petzold, K.; Stange, G.; Saleh, L.; Edenhofer, I.; Segerer, S.; Biber, J.; et al. Renal expression of FGF23 and peripheral resistance to elevated FGF23 in rodent models of polycystic kidney disease. Kidney Int. 2014, 85, 1340-1350. [CrossRef] [PubMed]

36. Hassan, A.; Durlacher, K.; Silver, J.; Naveh-Many, T.; Levi, R. The fibroblast growth factor receptor mediates the increased FGF23 expression in acute and chronic uremia. Am. J. Physiol. Renal Physiol. 2016, 310, F217-F221. [CrossRef] [PubMed]

37. Faul, C.; Amaral, A.P.; Oskouei, B.; Hu, M.C.; Sloan, A.; Isakova, T.; Gutierrez, O.M.; Aguillon-Prada, R.; Lincoln, J.; Hare, J.M.; et al. FGF23 induces left ventricular hypertrophy. J. Clin. Investig. 2011, 121, 4393-4408. [CrossRef] [PubMed]

38. Chang, C.H.; Fan, P.C.; Chang, M.Y.; Tian, Y.C.; Hung, C.C.; Fang, J.T.; Yang, C.W.; Chen, Y.C. Acute kidney injury enhances outcome prediction ability of sequential organ failure assessment score in critically ill patients. PLoS ONE 2014, 9, e109649. [CrossRef] [PubMed]

(C) 2018 by the authors. Licensee MDPI, Basel, Switzerland. This article is an open access article distributed under the terms and conditions of the Creative Commons Attribution (CC BY) license (http:// creativecommons.org/licenses/by/4.0/). 